\title{
IMPROVING THE CARE OF DEINSTITUTIONALIZED PEOPLE WITH SEVERE MENTAL ILLNESS: AN EVIDENCE BRIEF
}

Introduction: National policies have highlighted deinstitutionalization, or shift in providing mental health care for individuals with severe mental illness (SMI) from institutional environments to the community. Progress varies among countries and it is recognized a global concern in low-middle countries. Inform decision makers about strategies and appropriate changes in the mental health policies, could be key factors for mental healthcare improvement. The aim of this study was to develop an evidence brief to subsidize actions and strategies to improve the care of people with SMI.

Methods: We used the Supporting Policy Relevant Reviews and Trials Tools for evidence-informed health policymaking as follow: 1) conducted capacity building workshops with relevant stakeholders; 2) identification of a priority policy issue within a Brazilian public health system; 3) promoted exchange among different policy-makers, researchers and stakeholders; 4) development of an evidence brief (EB) that addresses the problem of deinstitutionalization, and for it: search strategy was defined to retrieve relevant systematic reviews (SR) from databases focusing any type of care strategies for deinstitutionalized people treated in the community; methodological quality of included SR was assessed by tool AMSTAR; 5) organized regional deliberative dialogues with stakeholders as to embed decision makers tacit knowledge and experiences into the EB, crucial step towards moving evidence into action.

Results: Based on the best available scientific evidence we identified five options to address deinstitutionalization: i) Provide psycho educational programs; ii) Implement and monitor the practice of intensive case management; iii) Develop community mental health teams; iv) Promote assisted living; v) Strengthening interventions for acute psychiatric episodes. Stigmas, shortage human resources for mental health care, limited political and budgetary support are the main barriers to implementation.

Conclusions: Engaging key stakeholders and developing EB to subsidize evidenceinformed policy making may lead Brazil to improving the care of patients with deinstitutionalized mental disorders.

Keywords: deinstitutionalization; evidence-informed policy; health policy; mental health; 